\title{
Knowing what you know: Intellectual humility and judgments of recognition memory ${ }^{2}$
}

\author{
Samantha A. Deffler *, Mark R. Leary, Rick H. Hoyle \\ Department of Psychology and Neuroscience, Duke University, Durham, NC 27708, United States
}

\section{A R T I C L E I N F O}

\section{Article history:}

Received 3 December 2015

Received in revised form 1 March 2016

Accepted 2 March 2016

Available online $\mathrm{xxxx}$

\section{Keywords:}

Intellectual humility

Recognition memory

Response bias

Sensitivity

\begin{abstract}
A B S T R A C T
This study examined the relationship between recognition memory and intellectual humility, the degree to which people recognize that their personal beliefs are fallible. Participants completed the General Intellectual Humility Scale, an incidental old/new recognition task, and a task that assessed the tendency to over-claim one's knowledge. Signal detection analyses showed that higher intellectual humility was associated with higher discriminability between old and new items, regardless of whether the items were congruent or incongruent with participants' own beliefs. However, intellectual humility was not related to response bias, indicating that intellectually arrogant people were not biased to claim that they knew everything. Together, the findings support a relationship between intellectual humility and performance on memory tasks, indicating that individual differences in intellectual humility may partly reflect how people process information and judge what they do and do not know.
\end{abstract}

(c) 2016 Elsevier Ltd. All rights reserved.

\section{Introduction}

"It's not what he doesn't know that bothers me; it's what he knows for sure that just ain't so."

-Vice-president Walter Mondale during a Presidential debate with Ronald Reagan.

People are not always good judges of the accuracy of their beliefs, knowledge, and memories, and like Ronald Reagan, sometimes "know" things to be true that are demonstrably incorrect (Harvey, 1997; Hoffrage, 2004). The meta-cognitive bias to have greater faith in one's beliefs than is warranted obviously compromises the quality of people's decisions and leads to misguided actions that are based on incorrect assumptions. Although the tendency to overestimate the accuracy of one's beliefs is widespread (Dunning, Johnson, Ehrlinger, \& Kruger, 2003; Fischhoff, Slovic, \& Lichtenstein, 1977), people differ in the degree to which they recognize that what they believe to be true might be incorrect. For example, people who are less knowledgeable in a domain are less able to assess the limits of their understanding than those who are more knowledgeable (Kruger \& Dunning, 1999).

Our interest in this project was in the relationship between intellectual humility and people's judgments of their own knowledge? Intellectual humility involves the recognition that one's beliefs are fallible and, thus, the degree to which people acknowledge that that which they

\footnotetext{
th We thank the John Templeton Foundation for supporting this research and Ashley Hawkins, Alysson Light, and Micah Lattanner for their help with data collection

* Corresponding author at: Department of Psychology and Neuroscience, Duke University, Box 90086, Durham, NC 27708, United States.

E-mail address: samantha.deffler@duke.edu (S.A. Deffler).
}

believe to be true may, in fact, be incorrect. People who are high in intellectual humility are attentive to limitations in the evidence for their beliefs and are aware that their ability to obtain and evaluate relevant information is limited. Holding such an epistemic stance may lead intellectually humble people to avoid unfounded confidence in their beliefs (McElroy et al., 2014). Indeed, intellectual humility has been characterized as an epistemic virtue that is needed for effective learning and endeavors in which people aspire to seek the truth, such as science (Baehr, 2011; Roberts \& Wood, 2007). Along these lines, Elder and Paul (2012) listed intellectual humility among a small set of intellectual competencies that should be fostered in educational settings.

Most discussions of intellectual humility assume, often implicitly, that these differences arise primarily from motivational factors. For example, people high in intellectual humility are higher in epistemic curiosity, openness, and need for cognition (Leary et al., 2016), characteristics that are associated with the motivation to seek information and think deeply about topics. Such an epistemic approach should expose intellectually humble people to information that demonstrates the complexity and ambiguity of many issues and encourage them to question the veracity of their beliefs. In contrast, people who are low in intellectual humility have a lower tolerance for ambiguity (Leary et al., 2016), which may motivate them to avoid information that might raise questions about their existing viewpoints. Low intellectual humility is also associated with the tendency to be threatened by what one doesn't know (Krumrei-Mancuso \& Rouse, 2016) and with the motive to defend one's ego in the face of ignorance, errors, disagreements, and other signs of one's intellectual shortcomings (Gregg, Sedikides, \& Gebauer, 2011; Sedikides \& Gregg, 2008). In contrast, people high in intellectual humility indicate that they enjoy finding out new 
information that differs from what they already think is true (Leary et al., 2016).

Although individual differences in intellectual humility may indeed have motivational underpinnings, our interest was in the possibility that these differences might also reflect differences in the metacognitive capacity to recognize what one does and does not know. People higher in intellectual humility are assumed to be better at recognizing what they do and do not know than people who are lower in intellectual humility (Elder \& Paul, 2012). However, such ability could reflect one or both of two distinct attributes-a higher ability to discriminate correct from incorrect information or a lower tendency, or bias, to assume that one knows things.

The present study tested these two possible explanations using two distinct procedures. The first involved an incidental old/new recognition task in which participants read statements about controversial issues that were congruent or incongruent with their beliefs and were later tested on their memory for these statements. By presenting statements that they had seen earlier with statements that had not been presented, we were able to examine participants' ability to recognize items that they had seen before. Using analyses based on signal detection theory (Snodgrass \& Corwin, 1988), we tested whether intellectual humility is related to either detection sensitivity or recognition bias.

Sensitivity (sometimes called discriminability) refers to the difficulty of distinguishing old (known) from new (unknown) items. Bias refers to the degree to which one response is more likely than another-the extent to which participants believe they did or did not see items regardless of their actual status. People high versus low in intellectual humility might differ on either or both of these parameters. That is, compared to people low in intellectual humility, highly humble people might more accurately distinguish what they know from what they don't, or be less likely to consistently think that they had seen all items.

Because we thought that intellectual humility might be related to the degree to which people process information with which they do versus do not agree differently, participants read some items that were consistent with their beliefs and other items that were contrary to their beliefs. That is, people who are high in intellectual humility may be more likely to think about sentiments that disagree with their own views than people who are low, which could contribute to their ability to discriminate new from old information.

In a second task, participants were asked to rate their familiarity with a number of people, historical events, and scientific concepts, some of which were real and some of which were bogus (Paulhus, Harms, Bruce, \& Lysy, 2003). As in the first task, we calculated both the sensitivity and bias of participants' claims, showing us the degree to which they accurately distinguished real from bogus targets and the degree to which they are biased to over-claim knowledge across the board. Intellectually arrogant people, who view their own knowledge as infallible, may show a response bias to claim that they know something, even when that information is bogus.

\section{Method}

\subsection{Participants}

The study was advertised in the local community via fliers and in the independent weekly magazine as part of a set of "studies on personality, opinions, and relationships" for which participants would be paid $\$ 40$ for $2.5 \mathrm{~h}$ of participation ( $\$ 10$ was paid for this study). The sample included 157 participants ( $51.6 \%$ female), ranging in age from 21 to 61 $(M=31.5, S D=8.12)$. Most participants were White $(54.8 \%)$, Black (21.9\%), or Asian (13.5\%). The sample was relatively well-educated, with $70.3 \%$ having obtained at least a Bachelor's degree. Two participants' data were removed before analysis; one was distracted during the task, and the other had difficulty comprehending instructions.

\subsection{Materials and measures}

\subsubsection{General Intellectual Humility Scale}

The General Intellectual Humility Scale is a 6-item measure of the degree to which people recognize that their personal beliefs might be wrong (Leary et al., 2016). Sample items include "I accept that my beliefs and attitudes may be wrong" and "In the face of conflicting evidence, I am open to changing my opinions," answered on 5-point scales anchored by not at all like me and very much like me. The General Intellectual Humility Scale has high inter-item reliability and strong construct and criterion-related validity. Scores from this study were sufficiently reliable $(\alpha=.80)$. Scores ranged from 13 to 30 , with a mean of 22.64 ( $S D=3.98)$. Scores are associated with characteristics that involve cognitive openness, such as low dogmatism, trait openness, epistemic curiosity, need for cognition, and tolerance of ambiguity. High scores are also related to more positive responses to people who hold different views and to people who change their positions (Leary et al., 2016).

\subsubsection{Beliefs about controversial topics}

Four controversial topics were selected: legalization of same sex marriage (the study was conducted 3 months before the U. S. Supreme Court's decision legalizing same-sex marriage on 6/26/2015), use of drones, legalization of marijuana, and implementation of the common core curriculum. Twenty attitude statements were written about each of these topics-10 sentences that argued in favor of each topic and 10 sentences that argued against each topic-based on Internet searches for pro and con viewpoints about each issue. Each sentence began with a clause indicating both the topic and the position being advocated, followed by a reason or explanation for the position. For example, a statement supporting same sex marriage would begin with the stem "Same sex marriage should be legalized...," followed by a reason such as "... because it would allow both parents to be legally recognized as parents of their children." From these sentences, four counterbalanced stimulus sets containing these sentences were created by random sorting. Each participant viewed one of the four sets of statements, which contained 40 of the 80 sentences. Of the sentences not presented with a set during study, half were used as "new" items in a subsequent recognition task.

\subsubsection{Over-claiming task}

The Over-claiming Questionnaire (Paulhus et al., 2003) measures participants' claims of knowledge regarding bogus topics (e.g., Jacques Worthington, Hamrick's Rebellion) relative to their claims of realworld knowledge (e.g., Susan B. Anthony, Mount Rushmore) to provide an index of their tendency to over-claim that they know things that they don't actually know. Here, participants rated 64 items, 40 of which were real and 24 of which were foils on a Likert scale from 1 (never heard of it) to 5 (very familiar with it).

\subsection{Procedure}

Before beginning the study, participants completed a battery of questionnaires that included the General Intellectual Humility Scale, indicated the highest level of education that they had attained (some high school, high school degree or GED, Associate's degree, Bachelor's degree [4 year college], Graduate or professional degree) and rated their views on four controversial topics (legalization of same sex marriage, use of drones, legalization of marijuana, and implementation of the common core curriculum) from 1 (strongly disagree) to 6 (strongly agree).

Participants were seated at a computer and told that they would read a list of beliefs about controversial topics. They were instructed to think about each sentence and to proceed at their own pace. Forty sentences ( 5 pro and 5 con statements for each topic) that reflected a subset of the 10 pro and 10 con statements for each of the four topics described earlier were presented one at a time in a random order. The time 
that participants spent reading the statements was recorded automatically. Participants were not told that they would later complete an old/ new recognition test later in the experiment.

After reading the sentences, participants completed the Overclaiming Questionnaire (OCQ). Because the OCQ also served as a filler task between presentation of the 40 attitude statements and the old/ new recognition task, we wanted to keep the delay between reading the sentences and completing the recognition task constant across participants; to do this, each OCQ item was presented for a maximum of $5 \mathrm{~s}$. Each topic (e.g., literature, history) was presented at random, and the items within each topic were also randomized. If the participant responded before the time limit expired, he or she viewed a blank screen for the remainder of the $5 \mathrm{~s}$ until the presentation of the next OCQ item. If no response was recorded within the allotted time, the next item was presented. Thus, all participants spent $5.33 \mathrm{~min}$ on the OCQ ( 64 items $\times 5 \mathrm{~s}$ per item).

After completing the OCQ, participants completed a surprise old/ new recognition task of the sentences they had read earlier. All of the 40 attitude sentences read previously were mixed with 20 new statements. For each of the 60 items, participants first indicated whether they thought that the sentence was "old" (seen previously in the study) or "new" (not seen previously during the study). After this designation, participants rated their confidence in their response on a 1 (not at all confident) to 7 (very confident) Likert scale.

\section{Results}

After describing how the data were prepared for analysis, the results are presented in two sections-one focusing on recognition memory for the attitude statements and the other on participants' tendency to overclaim knowledge.

\subsection{Data preparation}

For both the old/new recognition task and the OCQ, two measures were computed based on Signal Detection Theory (SDT; Green \& Swets, 1966; see Abdi, 2007 for a comprehensive overview) using formulas provided by Stanislaw and Todorov (1999). SDT is used to analyze data where participants must decide whether an item is known (the feeling of familiarity from that item is a correct signal) or unknown (any familiarity feeling from the item is noise). The first parameter of SDT, $d^{\prime}$, quantifies the sensitivity, or distance between signal and noise distributions, for each participant. (Alternatively, one can conceptualize $d^{\prime}$ as the difference between the familiarity signal and the noise signal for each person). Larger values of $d^{\prime}$ indicate that a participant was better able to distinguish between familiar items and unfamiliar items, compared to a participant with a smaller $d^{\prime}$, because the signal from previously-seen items is of a larger magnitude than the noise of unfamiliar new items.

The second SDT parameter, $c$, quantifies the participant's response bias, the tendency to respond a certain way regardless of whether an item is actually old (known) or new (unknown). In other words, $c$ reflects the participant's overall strategy when responding whether something is old or new. For $c$, negative values indicate a bias to respond that an item, regardless of its actual status, is old or familiar; positive values indicate a bias to respond that an item is new or unfamiliar.

\subsection{Old/new recognition task}

The General Linear Model program in SPSS was used to examine participants' accurate identification of the old and new attitude statements as reflected in $d^{\prime}$ and $c$ as a function of intellectual humility scores and whether a given statement was congruent or incongruent with a participant's views in a domain.

Intellectual humility predicted participants' sensitivity to the differences between old and new items $\left(d^{\prime}\right), b=.05,95 \% \mathrm{CI}[.007, .087]$,
$F(1,153)=5.59, p=.019, \eta^{2}=.04$, regardless of the statements' congruency with participants' beliefs. That is, participants who scored higher in intellectual humility more accurately distinguished old from new items. Congruence with participants' attitudes was not related to $d^{\prime}, F(1,153)=.07, p=.79$, nor was there an interaction between congruency and intellectual humility, $F(1153)=.06, p=.80$

Intellectual humility was not related to response bias $(c), F(1153)=$ $1.12, p=.29$, nor was there an effect of attitude congruency $F(1153)=$ $89, p=.35$, or an interaction, $F(1,153)=.55, p=.46$. Thus, participants higher in intellectual humility were better at discriminating between previously read sentences and new sentences, but intellectual humility was unrelated to an overall bias to say that all items were new or all items were old.

We considered that the relationship between intellectual humility and $d^{\prime}$ may reflect the amount of consideration that participants gave to each of the sentences; people who are high in intellectual humility, who are open to opinions different from their own, may spend more time considering evidence for and against a given issue. However, we found that intellectual humility was not related to the time that participants spent reading the sentences (calculated as the time spent reading each sentence divided by the number of words in the sentence to control for sentence length), $F(1,153)=2.16, p=.14$.

There was a significant main effect of attitude congruence on time spent reading the sentences, $F(1,153)=6.79, p=.010, \eta^{2}=.04$, that was qualified by a significant interaction between intellectual humility and the attitude-congruence of the sentence, $F(1153)=7.89, p=$ $.006, \eta^{2}=.05$. Follow-up analyses revealed that intellectual humility predicted time reading for sentences that were incongruent with participants' beliefs, $b=.5 .65,95 \% \mathrm{Cl}[.27,11.02], F(1,153)=4.31, p=.040$, with participants high in intellectual humility reading incongruent sentences for longer than participants lower in intellectual humility. Intellectual humility was not related to how long participants read congruent sentences, $b=1.71,95 \% \mathrm{CI}[-3.17,6.60], F(1,153)=.47$, $p=.49$.

Although participants who were higher in intellectual humility spent more time considering sentences that were incongruent with their views, the lack of an effect of sentence congruency on $d^{\prime}$ suggests that this differential reading time is not driving the relationship between intellectual humility and recognition. We conducted separate hierarchical regression analyses predicting $d^{\prime}$ for attitudecongruent and attitude-incongruent sentences from intellectual humility while controlling for reading time at Step 1. For both analyses, intellectual humility was significantly related to sensitivity $\left(d^{\prime}\right)$ : attitude-congruent, $\Delta R^{2}=.03, F_{\text {change }}(1,152)=4.18, p=.043$; attitude-incongruent, $\Delta R^{2}=.04, F_{\text {change }}(1,152)=4.80, p=.030$. The amount of time spent reading the sentences was not related to $d^{\prime}$ for either attitude-congruent or attitude-incongruent sentences, $b=<.001,95 \% \mathrm{CI}[-.001, .002] ; b=<.001,95 \% \mathrm{CI}[<-.001, .002]$, respectively. The interaction between intellectual humility and reading time was not significant in either model, attitude-congruent: $\Delta R^{2}=.00, F_{\text {change }}(1,151)=.08, p=.774$; attitude-incongruent: $\Delta R^{2}=.00, F_{\text {change }}(1,151)=.37, p=.546$. Thus, the relationship between intellectual humility and sensitivity to the difference between old and new items was not attributable to differences in reading time for attitude-congruent and attitude-incongruent sentences, nor did this relationship vary as a function of time spent reading the sentences.

\subsubsection{Confidence ratings}

As they completed the old/new recognition task, participants also rated their confidence that their identification of each sentence as old or new was correct. We expected that intellectual humility would be related to participants' confidence in their answers, particularly if those answers were incorrect. As when analyzing $d^{\prime}$ and $c$, we examined participants' confidence in their answers as a function of attitude-congruence, whether the answer was correct or incorrect, 
and intellectual humility. (Note that the degrees of freedom here differ; some participants did not have incorrect answers in some of the conditions, which are necessary to compute $d^{\prime}$ and $c$, so their data were not analyzed). The only effect was a significant interaction between answer correctness and intellectual humility, $F(1,144)=6.74, p=.010, \eta^{2}=$ .04 . For all other effects, $p s>.35$. To probe this interaction, simple regression analyses were conducted predicting confidence for correct and incorrect answers as a function of intellectual humility. Intellectual humility was negatively related to participants' confidence in their responses for incorrect answers, $b=-.05,95 \% \mathrm{CI}[-.099,-.004], F(1$, $152)=4.51, p=.035$, but not for correct answers, $b=.002,95 \% \mathrm{CI}$ $[-.029, .032], F(1,152)=.01, p=.914$. Participants who were high in intellectual humility were less confident in their incorrect answers than participants low in intellectual humility; intellectual humility was not related to confidence in correct answers.

\subsection{Over-claiming task}

Given that the OCQ responses were on a Likert scale rather than a dichotomous choice, they were analyzed according to the method utilized by Paulhus et al. (2003) in which scores are calculated for each of the cut-points on the Likert scale and then averaged. In SDT terms, using this approach with "4" being the cut-point, a real item rated as 4 or above is considered a hit (correctly responding that one knows a real item); the same item rated as a 3 or below is considered a miss (incorrectly responding that one does not know a real item). Conversely, a foil rated as a 4 or above is a false alarm (incorrectly responding that one knows a fake item); a foil rated as a 3 or below is a correct rejection (correctly responding that one does not know a fake item). The values of $d^{\prime}$ and $c$ are calculated based on these considerations. The same procedure is followed for cut-points of 5,3, and 2; the values of $d^{\prime}$ and $c$ are then averaged. See Paulhus et al. (2003) for more explanation on converting scale responses into binary responses to calculate $d^{\prime}$ and $c$.

Because participants low in intellectual humility assume that their knowledge and opinions are more correct than people high in intellectual humility do, they might be expected to claim that they are familiar with topics that are, in fact, bogus; if so, they would show a negative response bias compared to those high in intellectual humility. However, analysis of bias scores contradicted our expectations. The findings suggest no relationship between intellectual humility on response bias. A hierarchical multiple regression analysis with $c$ as the outcome variable was conducted with education level entered in the first step (as a proxy for general knowledge) and intellectual humility entered in the second step. Neither variable predicted $c$ on the over-claiming task (education level, $b=.03,95 \% \mathrm{CI}[-.019, .088], t(153)=1.26, p=.209$; intellectual humility, $b=-.002$, 95\% CI $[-.017, .012], t(153)=-.31, p=.758$.

However, intellectual humility did predict sensitivity to the difference between real items and foils. Intellectual humility predicted significant variance in $d^{\prime}, b=.03,95 \%$ CI $[.011, .053], F(1,153)=8.97, p=$ .003. This relationship remained even when education level was controlled for, $F(1,152)=4.20, p=.042$. For the model with both variables entered, both education level $(b=.22,95 \%$ CI [.149, .290], $t(152)=$ $\left.6.19, p<.001, s r^{2}=.19\right)$ and intellectual humility $(b=.02,95 \% \mathrm{CI}$ $[.001, .039], t(152)=2.05, p=.042, s r^{2}=.03$ ) accounted for participants' $d^{\prime}$ scores. Thus, although intellectual humility was not related to response bias, participants who were higher in intellectual humility more accurately discriminated between real and bogus items on the OCQ than participants who scored low. To say it differently, their beliefs about their own knowledge appeared to be more accurate.

\section{Discussion}

This study examined the relationship between intellectual humility and cognitive measures of recognition sensitivity $\left(d^{\prime}\right)$ and bias $(c)$ in two dissimilar tasks: an incidental old/new recognition task involving attitude statements and an over-claiming task that assessed participants' ratings of familiarity with real people, places, and events compared to plausible foils. On both tasks, higher intellectual humility was associated with higher $d^{\prime}$ scores. As noted, $d^{\prime}$ reflects the ability to tell the difference between old items (i.e., those encountered previously, whether in the experiment as with the old/new recognition task, or in the real world as with the over-claiming task) and new items (i.e., those not encountered previously), and it is calculated using the hit rate and the false alarm rate for each participant. Here, higher intellectual humility was associated with distinguishing more successfully between previously seen items (old/new task) or real facts (overclaiming task) versus new items or plausible foils, respectively. Even when education was taken into account as a proxy for general knowledge, intellectual humility was related to the ability to distinguish between real items and plausible foils on the OCQ. Our study focused on the general tendency to be intellectually humble, but recent work by Atir, Rosenzweig and Dunning (2015) suggested that self-perceived expertise (which could be a correlate of intellectual arrogance) in a domain, regardless of actual knowledge, predicts accuracy (calculated as hits minus false alarms) on the over-claiming task.

Critically, intellectual humility was not related to response bias (c), the tendency to respond "old" or "new" regardless of whether the item had been seen before. The lack of a relationship between intellectual humility and response bias was unexpected. One might assume that people low in intellectual humility would be more likely to respond that they knew things that they did not know than intellectually humble people (i.e., a bias to respond that all items were old or known; Dunning et al., 2003; Kruger \& Dunning, 1999). Interestingly, although they were not biased to think they knew things that they did not, intellectually arrogant participants were more confident in their incorrect answers on the old/new recognition task. This difference was not due to intellectually humble people being less confident overall, as it was not seen for confidence in correct answers. Put simply, people low in intellectual humility are more confident in answers that are wrong, possibly because low intellectual humility is linked to feeling threatened by being incorrect (Krumrei-Mancuso \& Rouse, 2016).

Intellectual humility was also related to how long participants spent reading sentences that were contrary to their beliefs. Participants who scored higher in intellectual humility spent more time reading attitude-incongruent sentences than participants lower in intellectual humility; however, they did not differ in reading time for attitudecongruent sentences. Given that intellectual humility involves openness to opinions counter to one's own (Leary et al., 2016), the fact that intellectually humble people considered attitude-incongruent sentences longer was expected. However, this differential reading time did not impact performance on the subsequent old/new recognition task, as the relationship between intellectual humility and $d^{\prime}$ remained when controlling for reading time.

These results, and the conclusions drawn from them, are not without limitations. First, our sample was comprised of relatively well-educated participants, who might be either more or less certain of what they know than less educated people. Either way, whether the results generalize to people who differ in education and intellectual humility is not known. In addition, although the results of the old/new recognition task were not moderated by participants' personal beliefs, we do not know whether people low versus high in intellectual humility might respond differently to topics that are more personally-relevant, important, or emotional.

\section{Conclusions}

Individual differences in intellectual humility are clearly associated with the cognitive processing of new information and people's metacognitive recognition of their own knowledge. During incidental learning and subsequent old/new recognition, intellectually humble people considered sentences counter to their own opinions for longer and also distinguished more successfully between sentences they had read 
previously and those they had not. When testing their ability to judge knowledge they had (or had not) encountered before, intellectually humble participants again outperformed intellectually arrogant ones, even when controlling for general knowledge (via education level). These findings suggest that individual differences in intellectual humility may partly reflect how people process information and judge what they do and do not know.

The effects demonstrated here may be due to differential attentional processing while learning new information; intellectually humble individuals may pay more attention to new information, and therefore perform better on judgments of recognition memory. Future research could test whether attention and other features of information processing differ as a function of intellectual humility. Do intellectually humble people hold more information in working memory, thereby allowing an easier simultaneous assessment of alternative views? Do they have stronger semantic associations between contradictory information? When studying cognition, we should take into account individual differences in how people process information, including differences in intellectual humility.

\section{References}

Abdi, H. (2007). Signal detection theory (SDT). Encyclopedia of measurement and statistics (pp. 886-889).

Atir, S., Rosenzweig, E., \& Dunning, D. (2015). When knowledge knows no bounds: Selfperceived expertise predicts claims of impossible knowledge. Psychological Science, $26,1295-1303$

Baehr, J. (2011). The Inquiring Mind: On Intellectual Virtues and Virtue Epistemology. New York: Oxford University Press.

Dunning, D., Johnson, K., Ehrlinger, J., \& Kruger, J. (2003). Why people fail to recognize their own incompetence. Current Directions in Psychological Science, 12, 83-87.

Elder, L., \& Paul, R. (2012). Critical thinking: Competency standards essential to the cultivation of intellectual skills, part 4. Journal of Developmental Education, 35, 30-31.
Fischhoff, B., Slovic, P., \& Lichtenstein, S. (1977). Knowing with certainty: The appropriateness of extreme confidence. Journal of Experimental Psychology: Human Perception and Performance, 3, 552-564.

Green, D. M., \& Swets, J. A. (1966). Signal detection theory and psychophysics. Oxford, England: John Wiley.

Gregg, A. P., Sedikides, C., \& Gebauer, J. E. (2011). Dynamics of identity: Between selfenhancement and self-assessment. In S. J. Schwartz, K. Luyckx, \& V. L. Vignoles (Eds.), Handbook of identity: theory and research. Vol. 1. (pp. 305-327). New York, NY: Springer.

Harvey, N. (1997). Confidence in judgment. Trends in Cognitive Sciences, 1, 78-82.

Hoffrage, U. (2004). Overconfidence. In R. Pohl (Ed.), Cognitive illusions: A handbook on fallacies and biases in thinking, judgment, and memory (pp. 235-254). Hove, East Sussex, UK: Psychology Press.

Kruger, J., \& Dunning, D. (1999). Unskilled and unaware of It: How difficulties in recognizing one's own incompetence lead to inflated self-assessments. Journal of Personality and Social Psychology, 77, 1121-1134.

Krumrei-Mancuso, E. J., \& Rouse, S. V. (2016). The development and validation of the comprehensive Intellectual Humility Scale. Journal of Personality Assessment, 98, 209-221.

Leary, M. R., Diebels, K. J., Davisson, E. K., Isherwood, J. C., Jongman-Sereno, K. P., Raimi, K. T., ... Hoyle, R. H. (2016). Cognitive and interpersonal features of intellectual humility. Manuscript under review Durham, NC: Duke University.

McElroy, S., Rice, K., Davis, D. E., Hook, J. N., Hill, P. C., Worthington, E. L., Jr., \& Van Tongeren, D. R. (2014). Intellectual humility: Scale development and theoretical elaborations in the context of religious leadership. Journal of Psychology and Theology, 42, 19-30.

Paulhus, D. L., Harms, P. D., Bruce, M. N., \& Lysy, D. C. (2003). The over-claiming technique: Measuring self-enhancement independent of ability. Journal of Personality and Social Psychology, 84, 890-904.

Roberts, R. C., \& Wood, W. J. (2007). Intellectual virtues: An essay in regulative epistemology. Oxford, UK: Clarendon.

Sedikides, C., \& Gregg, A. P. (2008). Self-enhancement: Food for thought. Perspectives on Psychological Science, 3, 102-116.

Snodgrass, J. G., \& Corwin, J. (1988). Pragmatics of measuring recognition memory: Applications to dementia and amnesia. Journal of Experimental Psychology: General, 117, 34.

Stanislaw, H., \& Todorov, N. (1999). Calculation of signal detection theory measures. Behavior Research Methods, Instruments, E Computers, 31, 137-149. 